\title{
Interférences
}

Ars scribendi

$10 \mid 2018$

Varia

\section{Le De Officiis et la pédagogie cicéronienne}

\section{Franck Colotte}

\section{(2) OpenEdition}

Journals

Édition électronique

URL : https://journals.openedition.org/interferences/6005

DOI : 10.4000/interferences.6005

ISSN : $1777-5485$

\section{Éditeur}

HiSoMA - Histoire et sources des Mondes antiques

\section{Référence électronique}

Franck Colotte, «Le De Officiis et la pédagogie cicéronienne », Interférences [En ligne], 10 | 2018, mis en ligne le 09 février 2018, consulté le 21 septembre 2021. URL : http://journals.openedition.org/ interferences/6005; DOI : https://doi.org/10.4000/interferences.6005

Ce document a été généré automatiquement le 21 septembre 2021

Tous droits réservés 


\title{
Le De Officiis et la pédagogie cicéronienne
}

\author{
Franck Colotte
}

\section{Cicéron entre tradition juridique et réévaluation philosophique}

1 Selon Aldo Schiavone, un Romain de l'époque de Cicéron, coutumier des lectures grecques et latines, à qui l'on demanderait quelles sont les strates les plus reculées ayant formé le droit de sa cité, désignerait d'un côté la tradition des pontifes, de l'autre les Douze Tables, « reliant ainsi, selon une vision courante à son époque, deux éléments que la mémoire culturelle aristocratique avait depuis des siècles intégrés en un canon unique: celui du ius ciuile, le majestueux et vénérable tronc de tous les ordres juridiques romains ${ }^{1} »$. De plus, comme le rappelle Anna Iacoboni dans son article « La crise de la res publica Romana et la reformulation du mos maiorum chez Cicéron », « le mos maiorum est une forme particulière de la consuetudo qui concerne notamment les maiores. [...] La tradition reçoit la légitimation de la uetustas et du consensus général, elle est renforcée par son application répétée. Ces facteurs sont nécessaires pour que celleci puisse s'imposer de manière plus vaste au sein de la communauté ${ }^{2}$. Le mos maiorum est transmis par tradition familiale et oralement. Les détenteurs de la tradition, c'està-dire les patriciens, la manipulent facilement en fonction de leurs propres intérêts et selon la situation politique du moment. La tradition a pu se transmettre surtout grâce à l'historiographie, et les premiers annalistes s'appuient sur cette dernière. L'historien britannique Peter Astbury Brunt (1917-2005) note au demeurant que l'activité littéraire est la prérogative des membres des groupes politiques dominants, à savoir les hommes les plus cultivés et les plus riches ${ }^{3}$.

2 Or, selon Riccardo Orestano (1909-1988), auteur de plusieurs essais portant sur le droit romain, les structures sociales qui constituent la coutume possèdent une portée plus étendue lorsque l'ordre-État et l'ordre-communauté coïncident. L'«ordre social» coïncide avec l'« ordre juridique » lorsque les structures sociales formées par les modes 
de vie des citoyens deviennent des structures de l'ordre de la communauté. Le consentement des membres des groupes politiques dominants fait que la coutume s'impose de manière plus forte au sein de l'ensemble de la communautét. Depuis longtemps, la cité a attribué au ius une fonction disciplinante détachée non seulement de la religion, mais aussi de la politique. Cette séparation a accentué la spécialisation technique d'un tel savoir, qui s'est greffé sur le tronc de la ritualité religieuse des origines, puis s'est sédimenté dans une activité de responsa exercée dans des situations à replacer dans le cadre d'une sorte de ritualisme laïcisé, parfaitement absorbé par la culture républicaine. On voit ainsi s'établir les difficiles protocoles d'une technique de contrôle social qui va connaître un succès durable et inégalé, ce que Cicéron comprend très rapidement ${ }^{5}$. Or, à l'époque tardo-républicaine, la classe dirigeante est politiquement très divisée. Si, dans le passé, l'homme romain avait pour but le bien public, en cette époque de crise, l'intérêt personnel se substitue au salut de l'État. À cela s'ajoute le fait que, comme le note Claudia Moatti, les citoyens, et notamment ceux qui constituent les groupes dominants, manquent de la conscience politique nécessaire pour exprimer une tradition commune ${ }^{6}$. La res publica est profondément déstabilisée sur le plan politique, aussi bien en son sein qu'en dehors des murs de la ville. La crise des coutumes traditionnelles romaines engendre une lutte intense et une déstabilisation politique forte : elle rend impossible cette transmission pour ainsi dire naturelle des valeurs de la cité. C. Moatti voit l'histoire intellectuelle du dernier siècle de la République romaine comme marquée par une révolution intellectuelle qu'elle place sous le signe de la Raison. Les élites de cette époque modifient leurs questionnements, leurs discours, leurs pratiques, tout en s'interrogeant sur l'identité romaine. Cette crise de la tradition, que l'on doit réinterpréter et réinventer, engendre notamment la découverte du droit naturel, la controverse philosophique, la critique de la superstition et de la divination, la remise en question de l'autorité, la construction de l'universel romain. C'est de même à Rome qu'a lieu pour la première fois ce qu'A. Schiavone décrit comme l'excarnation du préceptif, c'est-à-dire la dissociation de la fonction juridique, par son auto-isolement sous la forme d'une technique spécifique ${ }^{7}$. Loin d'un aléa historique n'intéressant que Rome, ce phénomène serait à l'origine de la constitution de la raison juridique en Occident, sur le mode d'une analytique autonome. Michèle Ducos, quant à elle, dans son essai Rome et le droit ${ }^{8}$, souligne la contribution essentielle à la philosophie du droit qu'apporte Cicéron, dans la mesure où il s'interroge sur la réalisation de la justice en s'attachant au rôle des tribunaux et de l'équité, et où il cherche à établir les fondements philosophiques du droit ${ }^{9}$. Avec le De re publica (54 av. J.-C.) puis le De legibus (52 av. J.-C.), l'Arpinate affirme l'existence d'une loi naturelle, conforme à la raison (Cicéron, Rep., III, 33) :

«Il existe certes une vraie loi, c'est la droite raison; elle est conforme à la nature, répandue chez tous les hommes; elle est immuable et éternelle; ses ordres appellent au devoir; ses interdictions détournent de la faute. [...] Bref, cette loi éternelle et immuable s'imposera à toutes les nations et à tous les temps, et un seul dieu commun à tous sera comme l'éducateur et le chef de tous ${ }^{10}$ ".

Dans le De legibus, il analyse les fondements et l'origine de cette loi avec encore plus de précision. Avant de spécifier quelles sont les lois les plus utiles à la communauté civique, il entend établir leur fondement, définir le juste véritable, et "puiser la connaissance du droit [...] au cœur de la philosophie » (ex intima philosophia hauriendam iuris disciplinam $)^{11}$. Il y montre les limites du droit positif, inscrit dans les textes, qui ne saurait à lui seul fonder une justice digne de ce nom ${ }^{12}$. Cette " loi véritable », commune aux dieux et aux hommes, prend, dans l'âme humaine, la forme de la vertu appelée 
prudentia (le discernement), qui permet de distinguer ce qui est juste et ce qui est injuste. Associé à une sorte d'instinct inné, le droit naturel concerne les relations entre les membres d'une même famille ou d'une même cité. Affection, respect, reconnaissance sont les vertus à travers lesquelles s'affirme son existence. Cela signifie que, pour chaque situation, il est une conduite, conforme à la justice, qui permet de rendre à chacun ce qui lui revient.

C'est dans ce contexte conceptuel servant de cadre et de prémisses au De officiis que Cicéron fait le pari de la philosophie, qu'il emploie non seulement pour sortir de la rigidité juridique romaine ${ }^{13}$, mais encore pour chercher à combiner (avec le De oratore et avant encore dans le De inuentione) le droit avec l'aequum et le bonum ${ }^{14}$ : «en un mot, c'est le problème de la place dans le droit romain de l'équité, à la fois juge et suppléante de la loi, écrite ou non, qui se pose ici ${ }^{15} »$. La question qui se pose consiste précisément à comprendre d'où Cicéron est parti pour cet emploi de la philosophie, à savoir de la rhétorique et de l'ars nécessaire à la rhétorique, c'est-à-dire le droit. Le traité des Topica (44 av. J.-C.) - contemporain du De officiis - témoigne de la corrélation entre droit et rhétorique: le fait que ce traité ait pour dédicataire le jurisconsulte romain Caius Trebatius Testa n'est pas simplement symbolique ou honorifique, mais revêt une dimension philosophique. Rédigées lors d'une traversée entre Vélie et Rhegium, les Topiques appartiennent au genre oratoire, quoique le manuel ait un sujet plus restreint : il s'agit pour Cicéron d'offrir un panorama des divers "lieux" qui fournissent les arguments, en les définissant par rapport à leurs équivalents grecs. Le domaine de la rhétorique, lié très étroitement, chez Cicéron, à celui de la philosophie ${ }^{16}$, est sans nul doute celui où l'influence de l'aristotélisme paraît la plus marquée. Toutefois, ces traces de l'aristotélisme dans les traités oratoires de Cicéron n'ont pas toutes la même valeur probante, surtout si l'on constate qu'à l'exception du titre le traité ne renvoie à rien, ou presque, des Topiques d'Aristote ${ }^{17}$. Il se trouve en effet que la rhétorique postaristotélicienne, à laquelle l'Arpinate a souvent puisé, s'est nourrie des théories d'Aristote, non sans les compléter ou les modifier à l'occasion, en fonction également du double projet cicéronien, s'agissant de l'homme, de réforme éthique, et s'agissant de l'État, de réforme juridico-politique. Nous pouvons en effet observer que, dans le monde de Cicéron, inspiré par la Nouvelle Académie, traversé par l'absence de conclusions nécessaires et par la recherche d'une créance fiable, les modalités principales ne sont pas tant le nécessaire, le possible, le contingent, l'impossible, le vrai ou le faux, que des paramètres opératoires épistémiques et déontiques tels que le permis, l'interdit, le plausible, le vraisemblable. Dans les Topiques, la production de la croyance ou de la confiance - fides, composante traditionnelle du mos maiorum que l'Arpinate revisite en sorte d'être l'artisan d'une élaboration philosophique consistant en la rencontre harmonieuse de ces traditions avec les mœurs et théories nouvelles inspirées par l'hellénisme - se situe dans un contexte argumentatif juridique, les procédures topiques permettant à l'auteur de construire un pont entre des preuves argumentatives et une croyance garantie impliquant un niveau épistémique. Cicéron aura ces catégories discursives et éthiques présentes à l'esprit en rédigeant son $D e$ officiis, ouvrage qui participe d'une même logique de clarification, de synthétisation et d'élargissement de son projet philosophique initié en pratique durant sa carrière d'avocat et élargi spéculativement par l'ensemble des considérations épistémiques et déontiques - sous-tendu par ce que nous pourrions appeler une inférence naturelle (au même titre que l'on parle de droit naturel) - que ce traité comporte. Ainsi considérée, l'expérience cicéronienne du droit et de la pratique de la philosophie en tant 
qu'exutoire du droit positif donnant une impulsion au jusnaturalisme et en tant que transformation morale de l'âme humaine revêt une dimension matricielle qui sera pleinement à l'œuvre dans le De officiis.

\section{Statuts de la philosophie, entre compensation et pédagogie}

En écrasant la conjuration de Catilina (63-62 av. J.-C.), Cicéron avait détourné de lui les démocrates, notamment le tribun Clodius. De plus, un excès de vanité imprudent le prive du soutien de Pompée, vainqueur de Mithridate, allié désormais, dans le premier triumvirat, à César et à Crassus. En 60 av. J.-C., il perd l'influence politique dont il jouissait en 63, quand il avait réalisé une coalition « centriste » (aristocrates modérés et chevaliers) tout en bénéficiant de quelques sympathies parmi les démocrates, qui lui sont retirées après sa répression de l'insurrection catiliniste et son attaque contre Clodius. Or, à peine les consuls de l'année 58 sont-ils entrés en fonction que Clodius présente un projet de loi selon les termes duquel serait banni quiconque aurait fait exécuter sans procès des citoyens romains - cette loi visait en particulier Cicéron ${ }^{18}$. Dans la nuit du 19 au 20 mars 58, celui-ci quitte Rome, se voyant condamné à l'exil en vertu d'un plébiscite, sans avoir été jugé. Il est frappé de "mort civile» (capitis deminutio), interdit aqua et igni, puni de la confiscation de ses biens. Durant les années suivantes, il perd progressivement de son pouvoir politique au point qu'à son retour de Cilicie en janvier 49, il retrouve Rome en proie à la guerre civile entre César et Pompée. Malgré une période de doute et d'irrésolution, il soutient Pompée et le parti gouvernemental contre César qui s'est mis dans l'illégalité en franchissant le Rubicon. Après la bataille de Pharsale qui signe la défaite de Pompée, Cicéron revient en Italie où César lui pardonne et lui permet de rentrer à Rome, mais, cette fois, il ne joue plus aucun rôle politique. À cela s'ajoutent, sur le plan personnel, son divorce d'avec Terentia (46 av. J.-C.) et le décès de sa fille Tullia (45 av. J.-C.).

Ce bref aperçu qui fait état de quelques épisodes marquants de son existence nous fait comprendre dans quelle mesure on peut placer, avec Matthew Fox, l'activité littéraire et rhétorico-philosophique de Cicéron sous le signe notamment de la " compensation ", en raison de sa marginalisation du monde politique et de la vie publique ${ }^{19}$. Dans les années 50 à 40 av. J.-C., on ne peut qu'admirer la prodigieuse activité de Cicéron, ce que M. Fox qualifie de «frénésie » d'écriture ( frenzy of production ${ }^{20} »$ ). En effet, durant les douze dernières années de sa vie (55-43 av. J.-C.), on ne dénombre pas moins de dix-huit ouvrages conservés traitant de rhétorique, de théorie politique, et surtout de philosophie - l'activité d'« écrivain philosophique ${ }^{21}$ ", selon l'expression d'Albert Yon, se concentrant en réalité sur deux ans (46-44 av. J.-C.). Dans une telle perspective, on est en droit de se demander quels rapports Cicéron entretient avec la philosophie et la rhétorique. Cette dernière lui sert de point de départ dans la mesure où éloquence et philosophie s'appellent et se complètent, comme il le souligne au début des Tusculanes (Cicéron, Tusc., I, IV-7) :

«[...] j'ai toujours estimé en effet que, en philosophie, l'idéal serait de pouvoir traiter les plus hauts problèmes dans une forme riche et brillante (copiose ornateque), et je me suis entraîné à cela avec tant d'ardeur que j'en suis venu à oser même faire des cours (scholas) à la façon des Grecs ${ }^{22}$ ». 
Qu'il s'agisse des Tusculanes ou du traité Du destin, entre autres, la méthode suivie par Cicéron consiste soit à présenter la discussion sous la forme de la disputatio à la manière des skholai des philosophes grecs - c'est le procédé de la Nouvelle Académie, celui de Carnéade -, soit à adopter la forme de l'exposé continu (dans le De officiis par ex.). Au début du De officiis, Cicéron assure à son fils Marcus que, s'agissant de la «science philosophique" (philosophandi scientia), la même préoccupation de puissance (uis dicendi) et d'adéquation oratoires l'anime (Cicéron, Off., I, I-2) :

«[...] pour la science philosophique, je le cède à beaucoup d'autres, mais en ce qui concerne proprement l'orateur, l'art de parler de façon appropriée, précise, ornée (apte, distincte, ornate dicere), parce que j'ai passé ma vie à cette étude il me semble que, si je m'attribuais ce mérite, je le revendique en quelque sorte suivant mon bon droit ${ }^{23} »$.

Cicéron veut donc enseigner à son fils Marcus une belle langue, c'est-à-dire sa langue, qu'il a employée pour faire connaître aux Romains la philosophie grecque.

Tel est en effet le projet de l'Arpinate : introduire la philosophie à un lectorat romain, servir de médiateur entre la philosophie grecque et le public romain, et ce, dans le même esprit qu'il confesse, dans son traité De optimo genere oratorum, avoir traduit les orateurs grecs Eschine et Démosthène (Cicéron, Opt. Gen., V-14) :

«[...] et je les ai traduits non en interprète, mais en orateur, avec la même présentation des idées et des figures, en adaptant les mots à notre propre langue (uerbis ad nostram consuetudinem aptis) ${ }^{24} \ggg$.

Comme le souligne également $\mathrm{M}$. Fox qui rappelle à plusieurs reprises la complexité de sa production philosophique, il s'agit pour Cicéron de se faire l'adaptateur, le « réécrivain ${ }^{25}$ » de la tradition philosophique grecque qui l'avait nourri et formé durant ses propres études ${ }^{26}$, et qui doit être adaptée aux Romains en prise avec les transformations multidimensionnelles de l'époque tardo-républicaine ${ }^{27}$. Cette adaptation aux réalités conceptuelles romaines, balancée entre théorie et pratique, plutôt que d'opérer un quelconque contrôle sur les impulsions naturelles et sur les émotions, constitue une exhortation à faire de la philosophie. Cicéron est habité par la conviction que la philosophie pourrait faire la différence quand il s'agit du fonctionnement de Rome en tant qu'État et en tant que société28. En s'appuyant sur un passage du De re publica, M. Fox rappelle en substance que, s'agissant de la uirtus, l'ars (en tant que technique) n'est pas suffisante, mais que l'essentiel de la vertu consiste en son application (uirtus in usu sui tota posita est ${ }^{29}$ ), son application la plus élevée étant le gouvernement de la cité. Il ne s'agit pas pour Cicéron d'imiter les philosophes (isti) qui, enseignant dans les écoles entre les murs d'une salle (in angulis), à l'écart des affaires publiques, s'occupent des mêmes questions mais parlent au lieu d'agir. La défense de la philosophie est par ailleurs un thème constant qui reflète la position de Cicéron en tant qu'homo nouus pour qui la culture grecque constitue le seul capital symbolique qu'il pouvait acquérir ${ }^{30}$, le caractère unique de la production philosophique de Cicéron résidant dans l'interaction entre son engagement profond pour la philosophie et un certain scepticisme quant à ses ramifications pratiques. Elle est un refuge contre les tempêtes auxquelles il a dû faire face, un substitut du gouvernement de l'État (philosophiam nobis pro rei publicae procuratione substitutam ${ }^{31}$ ). À cela s'ajoute le fait que la philosophie est non seulement un acte de résistance destiné à préserver les valeurs de la res publica en état de déliquescence ${ }^{32}$, mais encore un acte pédagogique d'un intérêt général que Cicéron se plaît à souligner (Cicéron, Diu., II, II-4 à 6) :

«En effet, quel plus grand, quel meilleur service pourrais-je rendre à la République que d'instruire et de former la jeunesse (quam si docemus atque erudimus iuuentutem), 
surtout quand les mœurs et les temps actuels l'ont entrainée sur une pente si dangereuse que les efforts sont nécessaires pour la réfréner et pour l'arrêter? [...] Leur empressement à me lire stimule, de jour en jour, mon empressement à écrire [...] Et puis, quelle conquête magnifique, quelle gloire pour les Romains, que de ne pas avoir besoin, pour la philosophie, d'ouvrages grecs. J'obtiendrai sûrement ce résultat, si je vais au bout de mon projet $^{33}$.»

Cicéron érige-t-il pour autant sa production philosophique et sa propre personne en un modèle à suivre? Se sentant investi d'une mission de médiateur et d'intégrateur (sur le plan tant linguistique que conceptuel) de la culture philosophique grecque, l'Arpinate est-il un exemple de professeur pour son fils Marcus et pour les jeunes générations qui lisent ses écrits avec zèle (studium) ${ }^{34}$ ? Influencé par le néo-académisme qui refusait tout dogmatisme, Cicéron, dans son essai sur La Nature des dieux, énonce clairement sa posture non autoritaire dans la pratique de la philosophie (Cicéron, N.D., I, V-10) :

«[...] dans une discussion philosophique, on doit accorder de l'importance aux arguments fournis par la raison bien plus qu'à l'autorité. De plus, l'autorité de ceux qui se posent en maîtres nuit bien souvent à ceux qui veulent apprendre ${ }^{35}$ ".

La ratio prime donc sur l'auctoritas (celle qui, concernant Cicéron, connaît un fort déclin de 62 à 51 av. J.-C.) : cette perspective est pleinement à l'œuvre dans le De officiis, et ce, d'autant plus que ce traité est placé sous le signe à la fois des échanges de devoirs (officia) et de bienfaits (beneficia), et des biens dans un contexte où sont présentées les activités manuelles et commerciales, comme le montre l'étude de Claire FeuvrierPrevotat portant sur les occurrences de «donner et recevoir» dans cet ouvrage ${ }^{36}$. Au demeurant, quels sont les courants qui orientent l'enseignement philosophique de Cicéron dispensé dans son De officiis? Dans le De natura deorum, il rappelle que ses maitres furent les stoïciens Diodote et Posidonius, ainsi que les académiciens Philon de Larissa et Antiochus d'Ascalon ${ }^{37}$. Cicéron se revendique comme garant de la méthode issue de Socrate (renouvelée par Arcésilas et affermie par Carnéade) consistant à parler " "pour et contre" les philosophes pour tenter de découvrir la vérité, qu'on ne peut prétendre atteindre avec certitude mais qu'on appréhende assez dans quelques représentations pour les juger "probables ${ }^{38}$ " ". Cicéron, en s'adressant à son fils Marcus, ne s'érige pas en modèle dogmatique, mais en chercheur de probabilités morales (dans la conduite d'un État ou d'un individu) envisagées à l'aune de courants philosophiques différents et sous-tendues par une méthode éducative fondée sur l'idée de faire accepter un objet et un comportement nouveaux à Rome. D'où lui vient cette approche? Rappelons que, pour Cicéron, la philosophia et les litterae l'ont aidé à se construire un rôle (persona) dans la citée ${ }^{39}$. L'Arpinate n'aura de cesse de rappeler que son zèle (studium) pour la philosophia et les litterae remonte à son enfance ${ }^{40}$. Il est par conséquent compréhensible que ce zèle se retrouve dans le De officiis dans lequel, après avoir présenté l'organisation générale de son sujet, articulée, on le sait, sur l'honestum (Livre I), sur l'utile (Livre II), et sur leur confrontation (Livre III), il étudie les composantes de l'honestum et les devoirs qui leur correspondent. L'option préférentielle en faveur du stoïcisme - que manifeste Cicéron - ne doit pas être interprétée comme un renoncement au scepticisme qu'il tient de son affiliation à l'Académie, mais doit s'entendre comme la mise en œuvre de cette liberté de jugement qui caractérise le philosophe platonicien qu'est Cicéron. 


\section{De officiis : enjeux et perspectives}

8 Texte au demeurant complexe, qui a donné lieu à un très grand nombre d'interprétations ${ }^{41}$, le De officiis a longtemps été perçu comme un code moral à valeur quasiment universelle ou comme un manuel de politologie à l'usage des dirigeants politiques ${ }^{42}$. De façon générale, le De officiis est considéré essentiellement comme un ouvrage de philosophie, très fortement marqué par le stoïcisme ${ }^{43}$. Rédigé à l'automne 44 av. J.-C. (derniers jours d'octobre-novembre ${ }^{44}$ ), ce traité est un ouvrage à la fois de circonstances (celles liées aux troubles politiques, sociaux et militaires qui caractérisent la période tardo-républicaine) et de continuation (inscrite dans le sillon philosophique de Panétius ${ }^{45}$ ). Cet ouvrage trouve son enracinement dans la situation précise de 44 av. J.-C., qui lui donne un réel caractère politique, presque militant, en sorte que l'on pourrait voir, avec Emilio Gabba, dans le De Officiis la quintessence du credo politique de Cicéron ${ }^{46}$. Les tensions extrêmes entre Cicéron et Antoine, que sépare notamment une vision de l'État diamétralement opposée, constituent le contexte politique dans lequel le traité du De officiis a été rédigé. Bien qu'il ne soit pas "d'inspiration soudaine ${ }^{47}$ » dans la mesure où il constitue le couronnement d'une ligne de pensée très ancienne ayant inspiré à son auteur toute sa carrière politique, cet ouvrage se caractérise par la rapidité de sa rédaction. Il ne s'agit pas, comme le relève Daniel Auverlot, d'un «travail accompli et mûrement ciselée ${ }^{48}$ ». Cicéron, qui n'a pas de plan d'organisation préétabli, suit un modèle, essentiellement Panétius, dans les deux premiers livres du traité ; dans le troisième livre, toujours d'inspiration stoïcienne notamment sous l'influence de Posidonius -, Cicéron, privé de guide et de canevas, essaie de prolonger par lui-même l'enseignement de Panétius. L'entreprise de notre auteur, à la fois dans sa dimension de philosophe et d'homme politique, est en effet double : il s'agit d'une part de poursuivre et d'achever l'ouvrage de Panétius tout en conservant l'esprit dans lequel il a été rédigé, et d'autre part de produire une réflexion de nature politique cherchant les conditions de redressement de sa cité. Dans sa tentative d'ordonnancement des devoirs, Cicéron est animé d'une préoccupation majeure : rendre "l'ordre à la cité ${ }^{49}$ " qui en est privée. Le De officiis correspond au besoin impérieux qu'a certainement ressenti Cicéron d'abord de conceptualiser, de rationaliser le mouvant, le latent; ensuite, de prendre, par l'écriture (même produite dans la précipitation), du recul par rapport aux nombreux événements qu'il venait de vivre; enfin, d'élargir les principes de la tradition romaine aux dimensions de l'univers ${ }^{50}$. Il s'agit aussi pour lui d'ordonner l'instable, le fluctuant, dans l'idée qu'harmonie entre citoyens et harmonie intérieure fondée sur des choix éthiques conscients et conformes à la vertu et à la justice sont deux faces complémentaires d'un même processus fondamental de réforme visant non seulement au "consensus » garant de toute action politique couronnée de succès ${ }^{51}$ - mais encore à la concordia, c'est-à-dire à l'harmonie intérieure de l'âme, condition du "consensus " ou son équivalent sur le plan éthique. Reprenant l'idée défendue par Cicéron selon laquelle la philosophie est la "culture de l'âme ${ }^{52}$ ", Philippe Rousselot ${ }^{53}$ relève que "cet effort intérieur est inséparable d'une recherche de la vérité en commun, d'une pensée en mouvement, en lutte contre elle-même, au risque de la contradiction et de l'indécision. L'exercice collectif du raisonnement philosophique est à l'image de la conviction que la pensée philosophique doit se mettre au service non des cénacles élitistes mais au service d'une cause élargie, celle de la société et celle de l'action ${ }^{54} »$. Un tel idéal montre à quel point l'Arpinate veut placer la philosophie au centre d'un modèle culturel 
romain, modèle qu'il échafauda patiemment tout au long de sa vie et dans lequel s'unissent le goût de la spéculation et le sens de l'action. Cicéron, qui, selon l'expression de Carlos Lévy, a décidé depuis 45 av. J.-C. de devenir le «professeur de philosophie du peuple romain ${ }^{55}$ ", se fait également pédagogue, notamment dans la mesure où son traité est dédié à son fils Marcus (ou, selon Maurice Testard, à un jeune homme qui a presque le même âge que Marcus, le jeune Octave, héritier de Jules (ésar ${ }^{56}$ ), alors étudiant à Athènes sous la direction du philosophe péripatéticien $\mathrm{Cratippe}^{57}$, et, de manière plus générale, est proposé à la formation éthique et politique de tous les jeunes Romains ${ }^{58}$.

\section{Une démarche pédagogique composite}

9 Dans le De officiis, Cicéron, avec les stoïciens dont il prend la suite, ne présente pas une morale « au rabais ", qui serait celle du grand nombre, pour l'opposer à une quelconque morale des purs, réservée aux sages. Il n'est pas question d'une éthique à deux temps, mais seulement d'une " conscience aiguë et lucide de la nature d'une "action droite" au sens strict ${ }^{59}$ ». En quoi consiste le devoir? Que doit faire l'homme? Que lui incombe-t-il de faire, de dire et de penser en tant qu'il est un homme ayant embrassé un choix de vie plutôt qu'un autre? Selon Christelle Veillard, pour Panétius, le devoir est «ce que la raison prescrit de faire, non pas la raison de n'importe qui, mais la raison de qui trouve ses principes d'action dans l'idée même de vertu humaine. Loin de proposer une morale de second ordre, une morale moyenne d'homme ordinaire, Panétius avance le modèle de l'homme accompli, c'est-à-dire du gentilhomme: un homme savant, magnanime, ordonné et mesuré, paraissant ce qu'il est, affable et bienfaisant pour tous les autres hommes ${ }^{60}$ ». De même, dans la théorie des «rôles » (personae) qu'expose Cicéron (I, 105 sq.), on comprend qu'il n'y a pas de morale rigide à sens unique. Il y a des actions appropriées à tous les hommes, mais d'autres qui dépendent de ces divers facteurs irréductibles à toute généralisation. C'est cette notion qui caractérise fondamentalement la nature du devoir : à condition d'agir en conformité avec chacun des «rôles", on peut effectivement fournir un justificatif rationnel "probable» expliquant pourquoi on a agi comme on l'a fait (I, 8). Ainsi que le rappelle Stéphane Mercier, "le devoir n'est donc pas affaire de transcendance, mais d'immanence, puisqu'il s'impose à l'agent en fonction de la nature qui est la sienne, et non d'après l'arbitraire d'un ordre extérieur. En ce sens, le devoir n'est pas une contrainte, mais la traduction, en actes, de l'autonomie d'un sujet agissant en conformité avec lui-même ${ }^{61}$ ».

Qu'en est-il, dans ces conditions, de la réalisation de la sagesse dans ce monde ? Rien ne s'y oppose en principe puisque la raison humaine ne présente pas de différence essentielle par rapport à la raison universelle. Il n'y a pas de devoirs du sage qui définiraient une "morale des purs", dont les critères seraient plus élevés que ceux d'une éthique du commun. Pour les uns comme pour les autres, il s'agit d'une seule et même morale, dont les applications ne se distinguent que parce les individus se distinguent entre eux, et que les circonstances dans lesquelles ils se trouvent ainsi que leurs choix de vie diffèrent. L'intention du sage et la qualité de sa résolution font que le devoir qu'il accomplit est «parfait». Tout insensé, s'il est de bonne volonté, n'est que l'ébauche du tableau parfait qu'est le sage, mais il n'est pas à dédaigner pour cela dans la mesure où l'esquisse peut s'embellir et se préciser sur la voie de l'honnête, lequel 
s'identifie avec le véritable utile. Comme Cicéron l'avait déjà souligné dans son $D e$ Inuentione ${ }^{62}$, une force innée a fait pénétrer en nous certaines notions, comme la religion, la pietas, la reconnaissance, le désir de vengeance, le respect, la vérité. Le droit naturel (naturae ius) se compose ainsi d'un ensemble de vertus sociales qui régissent les relations entre les hommes, et prescrivent de maintenir entre eux une égalité proportionnelle ce qui lui est dû, tout en respectant les impératifs de l'utilité commune, ce que Cicéron appelle l'aequum et le bonum. Parmi ces vertus (officia), dont tous les hommes ont reçu l'empreinte, figurent la bonne foi (fides $\left.{ }^{63}\right)$, le respect de la parole donnée, l'obligation de ne pas nuire, celle de respecter la propriété d'autrui ${ }^{64}$. Par ailleurs, comme le rappelle Michèle Ducos, c'est de la conception stoïcienne que se réclame Cicéron, à cette différence cependant qu'en bon Romain, il semble conférer au droit naturel à la fois une importance plus grande et une tournure plus juridique que ne l'avaient fait les philosophes du Portique ${ }^{65}$.

11 À cela s'ajoute que Cicéron transforme deux notions grecques distinctes, l'eulogon (terme commun aux stoïciens et à Arcésilas) et le pithanon (critère de l'action de Carnéade), en les unifiant par l'emploi d'un terme unique, probabilis ${ }^{66}$. Ce terme, qui signifie originellement «ce qui est digne d'être approuvé», "ce qui peut être approuvé », "ce qui est démontrable », en est arrivé à connoter la "probabilité, c'està-dire une notion statistique plus ou moins objective ${ }^{67}$ ». Carlos Lévy, dans son Cicero Academicus, rappelle que Cicéron, traduisant par le même terme probabile les deux termes grecs distincts eulogon et pithanon, emploie également le terme uerisimile ${ }^{68}$. La confusion qui en découle aurait pu être évitée si Cicéron n'avait pas pratiqué cette traduction alternative. Or, que signifie le concept de pithanon de Carnéade? Le pithanon carnéadien n'a pas pour soubassement la confiance absolue dans la Providence. Il résulte, à en croire Sextus, de la nécessité de donner un sens à l'action dans un monde d'incertitude ${ }^{69}$. Carnéade propose que l'on se fie pour agir au sentiment de vérité que donnent certaines représentations, mais sans pour autant en tirer des conclusions quant à leur conformité à la réalité. Le concept d'eulogon, quant à lui, indique ce à quoi l'on ajoute foi, ce qui est crédible, ce qui provoque l'assentiment ${ }^{70}$. Or, quelle transformation conceptuelle Cicéron opère-t-il dans le De officiis? Ce dernier n'a pas traduit la définition de «l'action correcte » par Arcésilas, mais il a traduit plusieurs fois la définition zénonienne du kathekon; et, en traduisant ainsi, il omet la partie sur la conséquence ou la conformité à la nature (Cicéron, Off., I, 8) :

medium autem officium id esse dicunt quod cur factum sit ratio probabilis reddi possit ${ }^{71}$

«Ils disent que le convenable moyen est ce dont on peut donner une justification

probable de la raison pour laquelle on l'a fait ».

Dans son De finibus, Cicéron donne une définition encore plus explicite (Cicéron, Fin., III, 58) :

est autem officium, quod ita factum est, ut eius facti probabilis ratio reddi possit ${ }^{72}$

«le convenable est ce qui est accompli de telle sorte que l'on puisse en donner une justification probable».

Comme le souligne Jean-Baptiste Gourinat, «cette opération de simplification n'est certainement pas innocente de la part de Cicéron: elle lui permet de ramener la conception stoïcienne du kathekon à la définition du katorthoma chez Arcésilas, définition qui ne comprenait que cette partie ${ }^{73} »$. De plus, en traduisant le terme eulogos par probabilis, Cicéron supprime toute connotation «raisonnable» de la définition stoïcienne de l'action convenable. Par cette traduction, il ramène les deux définitions (celle de Zénon et celle d'Arcésilas) au critère d'action carnéadien, ce qui permet à J.-B. Gourinat de conclure: "Tant l'occultation d'Arcésilas que l'altération de la 
définition de Zénon sont des décisions assez radicales de la part de Cicéron ${ }^{74} »$. En ramenant les deux termes eulogon et pithanon au seul terme probabile (et par là-même les deux notions à une seule), Cicéron a donc bien innové : le probabilisme est bien une invention de l'Arpinate, mais sur un fond doctrinal stoïcien ${ }^{75}$. Cicéron, qui connaît également l'importance du critère pratique de l'eulogon chez Arcésilas, aboutit à une synthèse de ces principes dans la catégorie du probabile, mot qui fait référence « au sens commun, à la raison et, en définitive, à la vérité, même s'il n'en constitue qu'une approche incertaine ${ }^{76} »$, et qui peut être considéré comme synonyme de uerisimile. L'importance de ce concept pour l'orateur est capitale dans la mesure où, loin de permettre l'accès à une connaissance totalisante, le probabile constitue " un instrument pour réaliser la persuasion, car grâce à lui la parole - tenue pour ni fausse, car elle ressemble au vrai, ni complètement vraie - devient un moyen opérationnel s'adaptant à toute situation. Une fois perdu le statut de cachette d'une vérité éternelle qui doit être dénichée, elle déploie sa puissance dans l'orientation des décisions d'autrui ${ }^{77}$ ».

Ainsi, conformément à l'enseignement du médio-stoïcien Panétius, la démarche à la fois éducative et parénétique de Cicéron défend une morale pratique moyenne, renvoyant - comme le note François Prost - « la vérité parfaite de la sagesse stoïcienne au domaine de l'inatteignable", et permettant alors à l'homme de "réaliser de l'excellence à l'imperfection assumée de sa nature, dans un esprit platonicien et non plus stoïcien ${ }^{78}$ ». Dans la perspective d'une morale moyenne accessible permettant à la jeunesse romaine d'adhérer à son projet - et au-delà de la jeunesse, d'un "grand programme de formation des Romains à la philosophie ${ }^{79}$ ", Cicéron, si l'on peut dire, fait feu de tout bois (mais non sans cohérence) afin de combiner, dans une « volonté de pédagogie nationale ${ }^{80} "$, morale théorique et pratique. Pour ce faire, en homme politique, rhéteur et philosophe soucieux non seulement du message qu'il souhaite transmettre aux générations futures, mais encore des méthodes et démarches censées emporter l'adhésion de son lectorat (présent et futur), Cicéron emploie, voire instrumentalise - dans la perspective d'une (re)valorisation de la tradition romaine (mos maiorum) -, une pédagogie composite, constituée de plusieurs piliers qui sont autant de composantes sur lesquelles il appuie son projet philosophique à visée éducative qui s'adresse à l'homme ordinaire à qui il veut inculquer ses idées philosophiques par l'intermédiaire de la mosaïque conceptuelle qu'en tant que philosophe se réclamant de la Nouvelle Académie et aimant les stoïciens en vue d'une sagesse romaine pratique ${ }^{81}$, il a créée dans la perspective d'une pédagogie de l'action. Instrument au service de la persuasion, le probabile permet à la parole de devenir un moyen opérationnel s'adaptant à toute situation. Cet ambitieux projet conduira l'auteur du De officiis à élaborer toute une casuistique "pour peser, selon chaque circonstance, ce qui est vraiment conforme au Bien absolu ou ce qui lui ressemble vraiment ${ }^{82}$ » et, de cette manière, faire émerger la vérité à travers la confrontation de différentes positions (philosophiques) ${ }^{83}$.

\section{Une pédagogie de l'exemple}

Un autre aspect important de la pédagogie cicéronienne à l'œuvre dans le De officiis est constitué par le fait qu'elle s'appuie sur des exempla, ces derniers, qu'ils soient historiques ou personnels, ayant résonné bien au-delà de la vie de leur auteur. Henriette van der Blom note que, de façon générale, l'Arpinate non seulement s'appuie 
sur une tradition de figures exemplaires provenant du passé de Rome, mais encore en introduit d'autres, soulignant et renforçant ainsi leur exemplarité ${ }^{84}$. Dans le quatrième chapitre de sa monographie (« Literature, History, and Philosophy. The Example of the De re publica »), M. Fox, quant à lui, note que le rôle de l'anecdote historique dans la philosophie de Cicéron est de produire un discours philosophique qui ne s'éloigne jamais des tropes et des thèmes familiers. Or, pour un public romain, rien ne peut être plus familier que le passé glorieux de Rome ${ }^{85}$. Jean-Michel David, auteur d'un article sur l'exemplum historique dans les discours judiciaires de Cicéron, précise également que «l'exemplum, la petite histoire courte qui rappelle un fait passé de la vie d'un grand homme, joue donc un rôle important dans la stratégie de l'orateur. Il peut certes n'avoir pour fonction que de rappeler un précédent et justifier un raisonnement ${ }^{86} »$. Ajoutons que l'orateur doit faire preuve de vastes connaissances historiques, comme Cicéron l'affirme dans ses traités De legibus (III, 18) et De oratore (I, 18 ; I, 159 ; I, 201). En effet, les exempla qu'il en retire remplissent une fonction à la fois ornementale, morale - par leur valeur de modèle - et explicative, qui profite à l'efficacité du discours, par un processus analogique. Claudia Moatti rappelle ainsi que, «dans la cité romaine où la généalogie jouait un si grand rôle, le citoyen se devait de connaître quantité d'exempla, maitres de vérités et modèles de vertu, répertoire écouté, appris par cœur et transmis oralement. À l'image des discours antiques, organisés selon une structure latente où abondent références à des discours ou des procès passés, à des techniques oratoires différentes, à des exempla, la société romaine s'articulait tout entière sur une faculté de commémoration et de remémoration ${ }^{87} »$. Eugen Cizek, quant à lui, conclut son article sur la poétique cicéronienne en précisant que "Cicéron, qui lui-même écrivit peu d'ouvrages historiques, se préoccupa surtout des tâches d'une historiographie qui aurait dû perfectionner l'homme, certes l'homme romain, à la faveur d'une large mise en perspective des faits mémorables. À son avis, l'historiographie serait un art indispensable au droit public et privé, à tout sénateur en général et notamment à l'orateur. En fin de compte, l'historiographie doit offrir aux Romains des modèles d'une bonne démarche politique et d'une digne pratique des mœurs ${ }^{88} »$. Et d'ajouter pour conclure : «Partout Cicéron plaide vigoureusement pour une historiographie loyale, mais surtout moralisatrice et éducative ${ }^{89} »$.

S'ajoute à cela une autre question: de quelle manière Cicéron définit-il l'exemple?

Voici la définition qu'il en donne dans son De Inuentione (I, 49) :

Exemplum est, quod rem auctoritate aut casu alicuius hominis aut negotii confirmat aut infirmat. Horum exempla et descriptiones in praeceptis elocutionis cognoscentur ${ }^{90}$

"L'exemple est ce qui confirme ou infirme l'argument par l'autorité ou le sort d'un homme ou d'une affaire. On en examinera des exemples et des définitions avec les préceptes de l'élocution ».

On observe une définition similaire dans le traité des Topiques $^{91}$. Dans les deux cas, l'auteur traite des moyens qui permettent une persuasion rationnelle et efficace. Le passage du De Inuentione appartient au chapitre traitant de la confirmatio, et l'exemplum y est une partie du comparabile. Celui des Topiques porte, comme la quasi-totalité de l'ouvrage, sur les lieux de l'argumentation, et fait de l'exemplum une partie de la similitudo. L'exemplum appartient donc au champ du probare, mais pas seulement, dans la mesure où Cicéron le cite aussi parmi les moyens du pathétique et de l'émotion ${ }^{92}$. Par ailleurs, pour Thomas Guard, dans son article portant sur les exempla dans le De officiis, l'exemplum est «un outil démonstratif qui place l'argument sous l'autorité d'un précédent connu de tous comme le souligne Cicéron lui-même dans le De Inventione ${ }^{93}$ ». 
En qualité d'instrument suscitant l'émotion et le plaisir chez l'auditeur-lecteur, les nombreux exemples que fournit Cicéron non seulement sont investis d'un fort pouvoir argumentatif, mais encore revêtent une portée philosophique et morale propre à instruire la jeunesse romaine, et au-delà d'elle, la nation romaine. Parmi les exemples cités, une majorité se réfère à l'histoire romaine récente, notamment à César ainsi qu'à Cicéron lui-même ${ }^{94}$. Dans le De officiis, l'exemplum a une portée explicative en ce qu'il contribue à la démonstration de l'auteur : ce traité se fixe pour objectif de brosser le portrait du sage stoïcien confronté au débat entre l'utile et l'honestum en prenant comme exemple la figure d'Hercule qui devient, sous la plume de l'Arpinate, l'archétype du sage choisissant un mode de vie conforme aux obligations stoïciennes ${ }^{95}$. De plus, l'exemplum n'est pas une simple illustration, une simple ornementation, il offre souvent une solution concrète, technique et précise aux problèmes sociopolitiques que rencontrent les Romains, comme en témoigne l'exemple de Régulus justifiant la supériorité de l'honestum sur l'utile (où Cicéron anticipe même les critiques que l'on pourrait lui adresser $\left.{ }^{96}\right)$. Ainsi, la force des exempla cicéroniens réside dans le fait qu'ils constituent un redoutable moyen de persuasion, notamment auprès d'un public romain fortement attaché au mos maiorum : "Grand utilisateur d'exempla historiques, Cicéron définit pour sa part les fonctions de l'exemplum en deux types généraux: l'argumentation, qui établit la fides du discours, et l'ornementation, qui vise à l'agrément ${ }^{97}$ ".

Un autre exemple illustrant ces réflexions générales est constitué par le personnage de Caton l'Ancien (Cato Maior) que Cicéron mentionne dans neuf passages tout au long de son traités ${ }^{8}$. Comme le note Laure Hermand, «l'omniprésence de la figure de Caton dans les œuvres de Cicéron est mise en valeur par l'auteur lui-même qui charge les personnages de ses dialogues de la faire remarquer au lecteur (ou à l'auditeur) sous forme de plaisanterie ${ }^{99} »$. Dans les deux traités que l'auteure analyse (Brutus, dans lequel l'Arpinate brosse un portrait élogieux de Caton, et Cato Maior de Senectute, dans lequel c'est le personnage de Caton lui-même qui mène le dialogue), Cicéron accorde une place importante, voire centrale, à l'une des grandes figures de l'âge d'or de la République. De même, dans le De officiis, le personnage de Caton est mentionné par Cicéron dans trois passages remarquables (I, 36-37; II, 89; III, 16). D'abord, dans le cadre de la guerre juste (bellum iustum) : le Censeur donne des recommandations à son fils - mis en congé par le consul alors qu'il servait en Macédoine lors de la guerre contre Persée - dans une lettre qui lui est destinée ${ }^{100}$. Les recommandations qu'il lui adresse, relatives aux formes légales du serment que prête le soldat avant d'engager le combat contre l'ennemi, s'inscrivent, selon la formule d'Andrew Roy Dyck, dans une série d'exempla iustitiae in hostem a maioribus nostris constituta ${ }^{101}$ donnée par Cicéron afin de renforcer la puissance de persuasion de son propos. Le même Caton l'Ancien intervient également dans le passage où l'Arpinate développe une réflexion sur la comparaison des choses utiles (II, 89). Il n'hésite pas à donner la parole à Caton dans le cadre d'une anecdote (également mentionnée par Pline l'Ancien et Columelle) qui provient probablement de ses Apophthegmata, collection de paroles mémorables ayant valeur de maxime - ici sur la manière de déterminer les devoirs ${ }^{102}$. Enfin, dans le livre III, 16, Cicéron consacre un long développement aux officia media qu'il distingue des officia perfecta : il assouplit ainsi la doctrine stoïcienne et l'humanise en accordant une grande place à la notion de progrès ${ }^{103}$. Caton, au même titre que C. Laelius et les Sept Sages de la Grèce antique, n'est sage que dans la mesure où il pratique ces devoirs moyens, portant ainsi «certaines ressemblances et apparences de sages». Sous la 
plume de Cicéron, il incarne donc la possibilité d'un progrès sur la voie de la sagesse par la pratique des officia media. Ces trois exemples mettent en évidence la part d'idéologie - défendre la romanité contre un hellénisme excessif ? - et les valeurs - par exemple le respect du sacramentum - dont Caton l'Ancien est porteur. Possédant une fonction de patronage littéraire, le Censeur permet à Cicéron de situer sa réflexion philosophique dans la continuité du mos maiorum, qu'il adapte à ses visées argumentatives.

16 En outre, Cicéron dévoile le processus de fabrication et le fonctionnement de l' exemplum en expliquant sa théorie des personae. Dans son article sur «Le Portique et le concept de personne », Maximilian Forschner précise que, dans le De officiis (I, 107-121), le terme de persona joue un rôle central ${ }^{104}$. Ce traité de Cicéron suit, dans ses deux premiers livres, l'ouvrage perdu de Panétius Peri tou kathèkontos; il s'agit de répondre à la question de savoir quel comportement, au sein du monde, est approprié à l'homme individuel. La théorie qu'on a appelée « la théorie des quatre personae » y apparait dans un contexte plus spécifique dans la mesure où elle se trouve incluse dans un débat assez long consacré à ce qui, dans le comportement d'un homme, est decorum, c'est-à-dire convenable, séant, moralement beau et plaisant. Cicéron, à la suite de Panétius, distingue quatre perspectives qu'il ne faut pas perdre de vue si l'on se demande ce qu'il convient de faire (quid deceat) en chaque circonstance. Ce sont ces quatre perspectives directrices au sein de décisions pratiques à valeur morale qu'il qualifie de personae. M. Forschner mentionne également que l'équivalent grec de persona est prosôpon: le mot apparait pour la première fois chez Homère pour caractériser le visage et la face humaine (par ex. Od., XIX, 361 ; XX, 352). Une deuxième signification de prosôpon est le visage artificiel que l'homme se procure en revêtant un masque. Comme le rappelle Maurice Nédoncelle, ce terme désigne au premier chef le masque, puis également par extension (après Aristote et à l'époque hellénistique) le rôle, le personnage que l'acteur d'une pièce met en scène par ses paroles et ses actions ${ }^{105}$. Dans cette perspective, le pluriel personae se trouve toujours pris dans le contexte d'un sujet numériquement un, qui adopte et interprète les divers rôles et les relie pour former, avec plus ou moins de succès, une personnalité harmonieuse ${ }^{106}$. Le premier rôle offre la structure générale de ce tout harmonieux qui constitue la beauté morale d'une vie humaine. D'autre part, l'homme n'atteint cette harmonie pour lui-même que quand il réalise également la deuxième persona que lui propose la nature, un rôle qui lui est spécifique en tant qu'individu. En outre, par decorum, Cicéron entend la manifestation extérieure de l'excellence d'un homme, une sorte de beauté qui fait resplendir la vie de celui qui est moralement bon (I, 94-98). Elle se définit, sous une forme qui est celle que lui donne le stoïcisme ancien en général, par référence au concept d'aequabilitas uniuersae uitae, tum singularum actionum, c'est-à-dire d'une conformité de l'existence dans son ensemble comme du détail des actions (I, 111). Envisageant l'identité personnelle dans sa dimension pratique, Cicéron précise que l'on atteint le decorum d'autant plus aisément que l'on respecte la particularité de sa nature dans la conduite de sa vie. L'Arpinate ajoute l'idée suivante : que l'on atteigne ou non le decorum dépend d'un agir qui fait ou non droit à notre rôle d'être humain et à notre rôle d'individu particulier. Quant à la troisième persona, qui intervient dans ce cadre, elle a son contenu dans la position sociale et politique où nous mettent la contingence et les circonstances dans lesquelles nous nous trouvons. Cette position entraîne des manières de se conduire bien définies, appropriées et convenables ou, inversement, déplacées et inconvenantes. À cela s'ajoute enfin une quatrième persona, qui reprend le thème des choix de vie. Elle est 
d'une part la résultante d'une décision où la réflexion et la délibération préliminaires se règlent sur les autres personae; d'autre part, une fois établie, elle constitue un paramètre éthique supplémentaire ${ }^{107}$. Cette théorie des quatre personae nous permet de conclure, avec M. Forschner, que « le point central implicite de la thèse de Cicéron est que le decorum présuppose une unité du libre choix et du choix de la carrière extérieure. La condition que l'on décide de se donner à soi-même doit trouver une expression harmonieuse dans la préférence que l'on donne à un champ d'activité donné dans le monde ${ }^{108}$ ». Ainsi, cette théorie, qui évoque quatre données élémentaires qui forment la condicio humana de chaque individu, offre chez Cicéron les perspectives directrices permettant de répondre à la question de savoir quels sont les rôles et les activités déterminés par ces derniers qui garantissent à chaque individu d'accomplir son être dans le monde et avec autrui. L'Arpinate s'appuie donc sur une anthropologie sociale basée sur une théorie du moi relevant à la fois de la psychologie et de l'éthique. Il se concentre sur la perspective sociale de ce que nous sommes et pouvons être en tant qu'hommes, ainsi que sur la pensée du choix de vie, l'unité du caractère, de la personnalité et de l'estime de soi qu'elle implique.

\section{Une pédagogie de la vertu}

17 Dans son analyse éthico-politique du concept de gloire, Giovanna Garbarino rappelle à juste titre que l'amitié caractérise les rapports d'un individu cum paucis, et que la gloire caractérise ceux du citoyen cum multis. Ces deux notions se fondant principalement sur l'amour - selon l'idéal stoïcien de la philia comme fondement des rapports sociaux, joint à la confiance et à l'estime, sentiments qui sont réciproques dans le cas de l'amitié, mais qui fonctionnent en sens unique dans celui de la gloire (de la multitudo à l'individu qui émerge et s'affirme ${ }^{109}$ ). Tout l'enjeu du De officiis, qui définit notamment les officia de l'homme politique, consiste donc à analyser les modalités éthiques selon lesquelles la gloire peut influencer la multitudo, orienter ses idées et ses comportements. Or, dans le deuxième livre de son traité intitulé De inuentione, Cicéron définit ainsi le terme de gloria (Cicéron, Inu., II, 166) :

gloria est frequens de aliquo fama cum laude $e^{110}$

«la gloire est une réputation élogieuse largement répandue, concernant une personne ».

Cette définition pourrait s'identifier à la bona fama (eudoxia) des stoïciens, selon le propos de Caton le Jeune que l'on relève dans le troisième livre du De finibus (III, 57) :

de bona autem fama-quam enim appelant eudoxian, aptius est bonam famam hoc loco appelare quam gloriam ${ }^{111}$

"quant à la bonne renommée - cette expression convient mieux ici que "gloire" pour rendre ce que les stoïciens expriment par eudoxian ».

Cette définition ajoute donc à l'idée première de "notoriété112 » une qualité morale. Or, dans le De officiis, Cicéron rappelle à son fils Marcus que la gloire constitue à Rome un capital familial, un héritage, un patrimoine (imitandos esse majores ${ }^{113}$ ) dont la conservation et l'accroissement exigent un effort soutenu (Cicéron, Off., I, 78) :

Licet enim mihi, M. fili, apud te gloriari, ad quem et hereditas hujus gloriae et factorum imitatio pertinet ${ }^{114}$

«Il m'est permis en effet, Marcus mon fils, de me glorifier auprès de toi qu'intéressent et l'héritage de cette gloire et l'imitation de ces actes ». 
Une partie du deuxième livre du De officiis (II, 31-51 ${ }^{115}$ ) est consacrée au concept de gloire que Cicéron évoque d'abord sous l'angle de la gloire militaire (Cicéron, Off., II, 45) :

in qua multi apud majores nostros exstiterunt ${ }^{116}$

" et c'est ainsi que beaucoup, du temps de nos aïeux, se révélèrent "

tout en précisant qu'il ne faut entreprendre de guerre qu'en montrant qu'elle n'a d'autre but que la paix ${ }^{117}$. Cicéron oriente ensuite son fils et l'ensemble de la jeunesse romaine vers des formes de gloire plus pacifiques : la science juridique et l'éloquence, que l'Arpinate considère comme indispensables à un homme d'État ${ }^{118}$. soumettre à la droite raison, sous peine de dégénérer en maladie de l'âme telle que, par exemple, la cupiditén ${ }^{11}$. À cela s'ajoute l'idée que la gloire simulant la vertu est particulièrement condamnable, comme en témoigne le passage suivant (Cicéron, Off., II, 43) :

Quod si qui simulatione, et inani ostentatione, et ficto non modo sermone, sed etiam vultu, stabilem se gloriam consequi posse rentur, vehementer errant ${ }^{120}$.

«Si donc certains pensent pouvoir obtenir une gloire durable par la simulation et un vain étalage, par la feinte non seulement dans les propos mais aussi dans le visage, ils se trompent lourdement. »

En quoi consiste donc la « vraie " gloire?

Si quis id ageret ut, qualis haberi uellet, talis esset ${ }^{121}$

répond Cicéron, c'est-à-dire, au fond, à être ce que l'on veut paraître. L'Arpinate conclut son propos en rappelant que «qui donc voudra obtenir la vraie gloire de la justice, qu'il s'acquitte des devoirs de la justice » (tels que l'auteur les a exposés en I, 20-41). Il rattache ainsi le concept de justice à l'officium (qui se définit par ce dont on peut donner une justification probable) y relatif, signifiant par là que le sujet, pour agir conformément à la justice, doit se fonder sur les seules forces de son jugement l'action juste devant mériter son approbation, même s'il a conscience que son action éthique n'est pas parfaite. En définitive, la recherche de la gloire, telle qu'un jeune Romain peut l'envisager, est éclairée par un autre passage du De finibus (III, 56-57). Dans ce dernier, le personnage de Caton le Jeune, à la suite des stoïciens pour qui la gloire n'est ni un mal ni un bien, donc indifférente, est amené à promouvoir la «bonne renommée » (bona fama) dans la hiérarchie des indifférents préférables. La vraie gloire, gratifiée d'une valeur propre, figure, en plus de l'amour et de la bienfaisance, parmi les moyens privilégiés pour se concilier les hommes. Ainsi considérée, en quoi consiste la quintessence de la pédagogie pratiquée par Cicéron? À associer la gloire à la vertu, qui la suit comme son ombre (Cicéron, Tusc., I, 109):

Etsi enim nihil habet in se gloria cur expetatur, tamen uirtutem tamquam umbra sequitur ${ }^{122}$

«car, bien que la gloire ne renferme rien de très désirable, elle accompagne

cependant la vertu comme son ombre ».

$\mathrm{Au}$ final, il insiste sur la vertu à caractère civique que constitue la gloire (Cicéron, Phil., I, 29) :

Est autem gloria laus recte factorum magnorumque in rem publicam meritorum, quae cum optimi cujusque tum etiam multitudinis testimonio comprobatur ${ }^{123}$

«Or, la gloire est la louange qui s'attache aux belles actions et aux grands services rendus à la patrie, sanctionnée à la fois par le témoignage de l'élite et par celui de la multitude ».

En définitive, l'exhortation à une recherche raisonnée d'une gloire vertueuse revêt un caractère civique. Parénèse et appel au civisme s'appellent et se complètent en vue d'instruire son fils Marcus sur son métier à la fois d'homme (par une incitation à un 
questionnement sur soi et sur le monde, fondé sur une action éthique appropriée) et de citoyen (qui se réalise dans l'engagement politique), le concept de probabile donnant «une dynamique à cette tension en permettant d'articuler l'action dans la cité des hommes et la contemplation du monde ${ }^{124} »$.

\section{Une pédagogie du respect}

Le terme de curriculum désigne l'ensemble des situations pédagogiques vécues au cours d'un cursus donné. Le curriculum cicéronien véhicule, comme nous l'avons vu, des valeurs morales en s'appuyant sur une éducation aux valeurs, le De officiis étant souvent considéré comme un code moral à dimension quasiment universelle ${ }^{125}$ ou comme un manuel de politologie à l'usage des dirigeants politiques ${ }^{126}$. Or, pour Platon, la philosophie est l'éducation ${ }^{127}$. Dès lors, il reste à s'entendre sur ce que la philosophie peut représenter en termes de contenus d'enseignement, sur la construction d'un réseau de savoirs et de valeurs propres à être organisés didactiquement. Le but du modèle d'éducation cicéronien est ambitieux, comme en témoignent les quatre composantes de l'honestum : la connaissance ${ }^{128}$ du vrai (cognitio); les fondements de la communauté humaine (societas hominum); la grandeur d'âme (magnitudo animi); le convenable (decorum). De ces quatre composantes, c'est la deuxième - prioritaire sur toutes les autres aux yeux de Cicéron ${ }^{129}$ - qui établit le lien social. Le premier devoir correspondant à l'établissement de ce lien social est la iustitia (traduction du grec dikaiosunè ${ }^{130}$ ), qui rend possible l'échange des devoirs et des bienfaits. Rappelons avec Ella M. Atkins que, de façon générale, Cicéron a confiance dans le système juridique romain, plus particulièrement dans sa conformité à la justice naturelle et dans son efficacité131, qu'il s'exprime en qualité de rhéteur ${ }^{132}$ ou en tant que théoricien de la politique ${ }^{133}$. Or, la fonction primordiale de la justice, que Cicéron qualifie de domina et regina uirtutum ${ }^{134}$, est d'abord de ne nuire (nocere) à personne, c'est-à-dire en premier lieu de ne pas porter atteinte aux biens des personnes, à leur propriété - cette dernière constituant, comme le note Alain Michel, la première des libertés individuelles: «L'homme ne peut en rien spolier l'homme. La propriété se confond quelque peu avec l'intégrité, que garantit la cité ${ }^{135} \%$. Cette réflexion sur la propriété, en plus de représenter en soi l'une des bases de l'organisation de l'État romain ${ }^{136}$, constitue par ailleurs un des fondements de la réflexion politique de l'Arpinate ${ }^{137}$. Ce dernier ajoute également qu'une autre fonction importante de la justice est d'utiliser des biens communs comme des biens communs et des biens privés comme des biens privés ${ }^{138}$. C'est dans cette même perspective que, dans son traité De re publica (I, $27^{139}$ ), il légitime la propriété par l'usage qui en est fait selon la loi de la nature. En effet, pour Cicéron, savoir utiliser les biens mis à la disposition des hommes par la nature permet de se donner le droit de se les approprier en toute justice. Dans une telle perspective, quelle conception de l'État Cicéron veut-il transmettre à la jeunesse romaine? Pour ce dernier, ceux qui pratiquent la justice, c'est-à-dire ceux qui défendent la propriété et ceux qui en usent comme il se doit, sont ceux qu'il appelle les boni uiri, c'est-à-dire la classe dirigeante ${ }^{140}$ sur laquelle il fonde tous ses espoirs pour le rétablissement de la République chancelante qu'il a sous les yeux. En employant une telle formulation au sujet des boni uiri - iustitia in qua uirtutis splendor est maximus, ex qua uiri boni nominantur - l'Arpinate n'exhorte-t-il pas son fils, ainsi que toutes les jeunesses présentes et futures, à prendre ces boni uiri comme des modèles républicains? 
21 En définitive, se dessine en filigrane, à partir de ces données, une théorie de la liberté, à la fois individuelle et collective, que Cicéron fait reposer sur quelques principes clés qui sont résumés dans le livre III du De officiis (III, 23). Ce passage important montre que le but ultime du droit est le respect de l'humain par l'humain, que le lien social s'appuie précisément sur cette donnée et que, comme le souligne Alain Michel, « la nature de ce lien se résume par le respect des droits individuels ${ }^{141} »$. Au final, la forme d'humanitas ${ }^{142}$ que Cicéron tente ici d'inculquer à la jeunesse romaine (et à tous ses lecteurs postérieurs) est particulièrement porteuse dans la mesure où elle établit une équivalence étroite entre le devoir sociopolitique et le respect humain.

\section{Conclusion : Cicéron, entre pédagogie et anthropologie}

Le De officiis est à la fois l'œuvre d'un philosophe, soucieux d'insuffler à son lectorat romain une conscience aiguë de la nature d'une action droite en fonction de toutes les situations qui pourraient se présenter dans une vie d'homme et de citoyen, et celle d'un réformateur, soucieux d'adapter les concepts philosophiques grecs - dont il est luimême issu - à la société romaine de l'époque tardo-républicaine, en proie à une crise morale et politique sans précédent. Nourri et influencé par plusieurs courants philosophiques - en particulier le néo-académisme et le stoïcisme, qui constituent la réserve conceptuelle dans laquelle il puise au gré de ses démonstrations-, Cicéron nourrit un projet ambitieux pour son fils, pour la jeunesse et même pour la nation romaine à laquelle il souhaite ouvrir les portes d'une sagesse accessible au plus grand nombre. Pour ce faire, l'Arpinate, attentif à placer la philosophie au centre d'un modèle culturel romain, rédige en quelque sorte son testament philosophique synthétisant l'ensemble d'un parcours de vie et de réflexions. Le De officiis peut être considéré comme l'espace dialogique bi/polycéphale dans le cadre duquel sont exposés plusieurs principes clés qui fondent son éthique, son humanisme, et qui sous-tendent l'ensemble des conseils prodigués sur un mode pédagogique. Ces derniers sont destinés à aider les jeunes Romains (et à travers eux peut-être la jeunesse du monde entier) à se former comme il se doit - c'est-à-dire en trouvant une réponse "moyenne » à des questions existentielles qui combinent l'éthique et le droit, à la fois dans leur métier d'homme capable d'exercer son libre jugement et dans celui de citoyen respectueux d'autrui et de l'État. Dans le contexte sociopolitique dans lequel ce traité a été rédigé (exil, opposition à Marc Antoine), on comprend que l'Arpinate, au soir d'une carrière d'avocat et d'homme politique, au terme d'un long cheminement philosophique, soit soucieux de livrer, comme d'autres avant lui, son ktêma eis aei, selon l'expression consacrée de Thucydide, pour la postérité.

Cicéron, en qualité de pédagogue, est soucieux de convaincre, de transmettre aux générations futures la somme d'expériences et de réflexions à la fois épistémiques et déontiques qu'il a accumulées au fur et à mesure de ses lectures et de la rédaction de ses traités. Il évoque sa préoccupation d'une belle langue latine, non seulement comme valorisation de la romanité, mais encore comme instrument destiné à faire connaître aux Romains la philosophie grecque dont il adapte certains concepts clés afin de servir ses visées argumentatives. Qu'il s'agisse du concept du probabile - qui, dans une entreprise de simplification, synthétise l'eulogon stoïcien et le pithanon carnédien -, de la théorie des quatre personae - qui permet en définitive de se construire une identité 
personnelle dans sa dimension pratique -, de celui de gloire vertueuse ou des exempla significatifs (par ex. Caton l'Ancien) qui servent d'adjuvants argumentatifs, Cicéron a à cœur de convaincre qu'un devoir est la traduction, en actes, de l'autonomie d'un sujet agissant en conformité avec lui-même. Pour ce faire, éloigné de l'agitation de la vie publique et animé d'une frénésie d'écriture, il ressent le besoin impérieux de conceptualiser, de rationaliser le mouvant et le latent, de prendre du recul, et enfin d'élargir les principes de la tradition romaine aux dimensions de l'univers. Bien que, dans une perspective néo-académicienne, il ne se considère pas comme un modèle à suivre - ni dans sa vie personnelle, ni dans sa vie publique -, Cicéron adopte la posture $\mathrm{du}$ professeur de philosophie du peuple romain : il est un passeur, un adaptateur aux réalités romaines d'un ensemble conceptuel issu du monde grec qu'il s'emploie à traduire et à interpréter.

Ainsi considéré, le De officiis s'adresse à tous ceux qui - simples citoyens ou hommes publics - sont animés du besoin à la fois épistémique et déontique de savoir ce qu'ils doivent faire en chaque circonstance. Dans une perspective à la fois anthropologique et humaniste, l'Arpinate souhaite que le sujet, numériquement un, adopte et interprète tous les rôles et les relie pour former une personnalité harmonieuse. La pédagogie qu'il déploie dans son traité est à la fois multidimensionnelle et à géométrie variable : synonyme de parénèse et d'appel au civisme, elle se veut une incitation à un questionnement sur soi et sur le monde, fondé sur une action éthique appropriée. Soucieux de fournir des exempla édifiants et stimulants à l'individu comme au citoyen susceptible d'exercer des responsabilités publiques et/ou militaires, l'Arpinate cherche à construire, pour chacun, une identité personnelle qui puisse servir de tremplin vers une liberté non seulement individuelle, mais encore collective. Il parie à la fois sur la philosophie - par l'intermédiaire des officia media - et sur la classe dirigeante des boni uiri pour rétablir la République agonisante qu'il a sous les yeux. Ainsi, l'humanitas qu'il développe dans le De officiis est une disposition (mos) qui caractérise la sociabilité : fondamentalement, l'homme humanus, pour Cicéron, est celui que son éducation a rendu apte à la vie sociale. Or l'Arpinate y contribue en ce sens qu'il défend l'idée selon laquelle l'homme atteint le decorum - manifestation extérieure de son excellence d'autant plus facilement qu'il respecte la particularité de sa nature dans la conduite de sa vie. En ce sens, l'approche anthropologique qui sous-tend le De officiis consiste à savoir « doser" (temperare) l'humanitas en fonction des situations de vie auxquelles on est confronté. C'est ainsi que Cicéron revendique cette humanitas héritée des Grecs en l'adaptant au monde romain, en même temps qu'il revendique la mission de l'étendre partout.

\section{BIBLIOGRAPHIE}




\section{Textes anciens}

Cicéron, Les Devoirs, éd. et trad. par M. TESTARD, CUF, Paris, Les Belles Lettres, 1965-1970, 2 vol. Cicéron, Les Devoirs, texte établi par M. TESTARD ; introd., nouv. trad. et notes par S. MERCIER, Classiques en poche 113, Paris, Les Belles Lettres, 2014.

Cicéron, De la divination, introd., trad. et notes par J. KANY-TURPIN, G.F. 1076, Paris, Flammarion, 2004.

Cicéron, De l'invention, texte établi et trad. par G. ACHARD, CUF, Paris, Les Belles Lettres, 1994.

Cicéron, La Nature des dieux, trad. et commenté par C. AUVRAY-ASSAYAS, La Roue à livres 42, Paris, Les Belles Lettres, 2009.

Cicéron, De l'orateur. III, Livre III, texte établi par H. BORNECQUE et trad. par E. COURBAUD et H. BORNECQUE, CUF, Paris, Les Belles Lettres, 1930.

Cicéron, L'Orateur ; Du meilleur genre d'orateurs, texte établi et trad. par A. YON, CUF, Paris, Les Belles Lettres, 1964.

Cicéron, La République, texte établi et trad. par E. BRÉGUET, CUF, Paris, Les Belles Lettres, 1980, 2 vol.

Cicéron, Des termes extrêmes des biens et des maux. texte établi et trad. par J. MARTHA, CUF, Paris, Les Belles Lettres, 1929-1930, 2 vol. ; $3^{e}$ tirage de l'éd. revue et corr. par C. RAMBAUX, 2002, 2 vol.

Cicéron, Divisions de l'art oratoire ; Topiques, texte établi et trad. par H. BORNECQUE, CUF, Paris, Les Belles Lettres, 1924.

Cicéron, Discours. XIX, Philippiques I à IV, texte établi et trad. par A. BOULANGER et P. WUILLEUMIER, $2^{\mathrm{e}}$ tirage revu et corr., CUF, Paris, Les Belles Lettres, 1963.

Marcus Tullius Cicero, Topica, ed. with a translation, introd., and commentary by T. REINHARDT, Oxford Classical Monographs, Oxford, Oxford University Press, 2003.

Cicéron, Traité des lois, texte établi et trad. par G. DE PLINVAL, CUF, Paris, Les Belles Lettres, 1959.

Cicéron, Traité du destin, texte établi et trad. par A. YON, Paris, Les Belles Lettres, 1933.

Cicéron, Tusculanes. I, Livres I-II, texte établi par G. FOHLEN et trad. par J. HUMBERT, CUF, Paris, Les Belles Lettres, 1960.

Platon, Cuvres complètes, sous la dir. de L. BRISson, Paris, Flammarion, 2008.

\section{Études modernes}

ATKINS E. M. 1990, «Domina et regina virtutum : justice and societas in De Officiis », Phronesis 35, 3, p. 258-289.

AUVERLOT D. 1990, «Structure et sens du livre I du De officiis », L'Information littéraire 42, p. 3-9. AUVRAY-ASSAYAS C. 2006, Cicéron, Figures du savoir 37, Paris, Les Belles Lettres. BRUNT P. A. 1979, Conflits sociaux en République romaine, Textes à l'appui. Histoire classique, Paris, Maspero.

CIZEK E. 1988, « La poétique cicéronienne de l'histoire », BAGB 1, p. 16-25. 
Colotte F. 2014, « Le De Officiis de Cicéron : un manuel de vertu pratique ? », Etica \& Politica 16, 2, p. 341-359.

DAUBIGNEY A. 1976, Texte, politique, idéologie : Cicéron. Pour une analyse du système esclavagiste, le fonctionnement du texte cicéronien, Annales littéraires de l'Université de Besançon 187, Centre de recherches d'histoire ancienne 20, Paris, Les Belles Lettres.

DAVID J.-M. 1980, « Maiorum exempla sequi : l'exemplum historique dans les discours judiciaires de Cicéron », MEFRA 92, 1, p. 67-86.

Ducos M. 1984, Les Romains et la loi. Recherches sur les rapports de la philosophie grecque et de la tradition romaine à la fin de la République, Collection d'études anciennes, Paris, Les Belles Lettres.

- 1996, Rome et le droit, Références 526, Paris, Librairie générale française.

DYCK A. R. 1996, A Commentary on Cicero, De Officiis, Ann Arbor, University of Michigan Press.

ERNOUT A., MEILLET A. 1967, Dictionnaire étymologique de la langue latine. Histoire des mots, $4^{\mathrm{e}}$ éd., $2^{\mathrm{e}}$ tirage augm., Paris, Klincksieck.

FEDELI P. 1973, « Il 'De Officiis' di Cicerone. Problemi e atteggiamenti della critica moderna », ANRW, I, 4, p. 357-427.

FERRARY J.-L. 1990, « Quelques réflexions sur le De officiis », VL 117, p. 2-6.

FEUVRIER-PREVOTAT C. 1985, «Donner et recevoir : remarques sur les pratiques d'échanges dans le De officiis de Cicéron », DHA 11, p. 256-290.

FORSCHNER M. 2005, « Le Portique et le concept de personne », in G. ROMEYER DHERBEY (dir.), Les stoïciens, Bibliothèque d'histoire de la philosophie, Paris, Vrin, p. 293-317.

FOX M. 2007, Cicero's Philosophy of History, Oxford, Oxford University Press.

GABBA E. 1979, « Per un' interpretazione politica del De Officiis di Cicerone », RAL 34, p. 129-130.

GARBARINO G. 1980, «Il concetto etico-politico di gloria nel De officiis di Cicerone », in Tra Grecia e Roma. Temi antichi e metodologie moderne, Biblioteca internazionale di cultura 3, Rome, Istituto della Enciclopedia italiana, p. 197-204.

GARCEA A. 2008, «Consule ueritatem : Cicéron, Varron et un chapitre de l'histoire de la vérité à Rome », RMM 57, p. 93-110 [en ligne]. URL : https://www.cairn.info/revue-de-metaphysique-etde-morale-2008-1-page-93.htm [consulté le 4 janvier 2018] ; DOI : 10.3917/rmm.081.0093.

GOURINAT J.-B. 2016, « Cicéron fondateur du probabilisme ? Remarques sur l'emploi du terme probabilis chez Cicéron ", in P. GALAND, E. MALASPINA (éd.), Vérité et apparence. Mélanges en l'honneur de Carlos Lévy offerts par ses amis et ses disciples, Latinitates 8, Turnhout, Brepols, p. 257-268.

GRILLI A. 1975, «Cicerone tra Antioco e Panezio », Ciceroniana N.S. 2, Atti del II Colloqium Tullianum, 1974, p. 72-80.

GRIMAL P. 1989, « Le De officiis de Cicéron », VL 115, p. 2-9.

GUARD T. 2007, «Morale théorique et morale pratique : nature et signification des exempla dans le De officiis de Cicéron », VL 176, p. 50-62.

HABICHT C. 2013, Cicéron le politique, trad. fr. S. BLUNTZ, Paris, Les Belles Lettres.

HERMAND L. 2009, « Entre figure historique et construction littéraire : Caton l'Ancien chez Cicéron (Brutus et De senectute) » [en ligne]. URL : https://hal.archives-ouvertes.fr/hal-00365214/ document [consulté le 4 janvier 2018]. 
IACOBONI A. 2015, «La crise de la res publica et la reformulation du mos maiorum chez Cicéron », Camenulae 13 [en ligne]. URL : http://www.paris-sorbonne.fr/IMG/pdf/IACOBONIbat.pdf [consulté le 4 janvier 2018].

JAEGER W. 1964, Paideia, Paris, Gallimard.

LADMIRAL J.-R. 1994, Traduire : théorèmes pour la traduction, 2éd., Coll. Tel 246, Paris, Gallimard.

LÉVY C. 1989, « Le De officiis dans l'œuvre philosophique de Cicéron », VL 116, p. 11-16.

- 1992, Cicero Academicus. Recherches sur les Académiques et sur la philosophie cicéronienne, Coll. de l'École française de Rome 162, Rome, École française de Rome.

- 2003, « Y a-t-il quelqu'un derrière le masque ? À propos de la théorie des personae chez Cicéron », Itaca 19, p. 127-140.

-2008, «Cicéron, le moyen platonisme et la philosophie romaine : à propos de la naissance du concept latin de qualitas », RMM 57, p. 5-20 [en ligne]. URL : https://www.cairn.info/revue-demetaphysique-et-de-morale-2008-1-page-5.htm [consulté le 4 janvier 2018] ; DOI : 10.3917/rmm. 081.0005.

MARCHAL L. 1987, « L'histoire pour Cicéron », LEC 55, 1, p. 41-64.

MICHEL A. 1976, «Philosophie grecque et libertés individuelles dans le De Officiis de Cicéron », in La filosofia greca e il diritto romano. I, Colloquio italo-francese - Roma, 14-17 aprile 1973, Rome, Acc. Nazionale dei Lincei, p. 83-96.

- 2003, Les rapports de la rhétorique et de la philosophie dans l'œuvre de Cicéron. Recherches sur les fondements philosophiques de l'art de persuader, $2^{\mathrm{e}}$ éd., Bibliothèque d'études classiques 34 , Louvain, Peeters.

MOATTI C. 1988, « Tradition et raison chez Cicéron : l'émergence de la rationalité politique à la fin de la République romaine », MEFRA 100, p. 385-430 [en ligne]. URL : http://www.persee.fr/doc/ mefr_0223-5102_1988_num_100_1_1597 [consulté le 4 janvier 2018].

- 1997, La raison de Rome. Naissance de l'esprit critique à la fin de la République (II ${ }^{e}-I^{e r}$ siècle avant JésusChrist), Des travaux, Paris, Éd. du Seuil.

NARDUCCI E. 1989, « Una morale per la classe dirigente », in Modelli etici e società. Un'idea di Cicerone, Biblioteca di materiali e discussioni per l'analisi dei testi classici 7, Pise, Giardini, p. 111-115.

NEDONCELLE M. 1948, « Prosopon et persona dans l'Antiquité classique », RSR 22, p. 277-299.

NICOLET C. 1976, Le métier de citoyen dans la Rome républicaine, Bibliothèque des histoires, Paris, Gallimard.

ORESTANO R. 1967, I fatti di normazione nell'esperienza romana arcaica, Turin, G. Giappichelli.

PALLASSE M. 1945, Cicéron et les sources du droit, Annales de l'Université de Lyon 8, Paris, Librairie du Recueil Sirey.

POHLENZ M. 1934, Antikes Führertum. Cicero, De Officiis und das Lebensideal des Panaitios, Neue Wege zur Antike. Reihe 2, Interpretationen 3, Leipzig, Teubner.

PösCHL V. 1987, « Quelques principes fondamentaux de la politique de Cicéron », CRAI 131, 2, p. 340-350.

PROST F. 2008, « La philosophie cicéronienne de l'amitié dans le Laelius », RMM, p. 111-124. 
Rousselot P. 2012, « Cicéron philosophe », iPhilo [en ligne]. URL : http://iphilo.fr/2012/12/08/ ciceron-le-philosophe/\#commentaires [consulté le 4 janvier 2018].

SCHIAVONE A. 2008, Ius. L'invention du droit en Occident, L'Antiquité au présent, Paris, Belin [trad. fr. par G. et J. BOUfFARTIGUE de : Ius. L'invenzione del diritto in Occidente, Biblioteca di cultura storica 254, Turin, G. Einaudi, 2005].

TATAKIS B. N. 1931, Panétius de Rhodes : le fondateur du moyen stoïcisme. Sa vie et son ceuvre, Bibliothèque d'histoire de la philosophie, Paris, J. Vrin.

TESTARD M. 1962, « Le fils de Cicéron, destinataire du De Officiis », BAGB 2, p. 198-213.

VALENTE P. M. 1956, L'éthique stoïcienne chez Cicéron, Paris, Librairie Saint-Paul.

VAN DER BLOM H. 2010, Cicero's Role Models. The Political Strategy of a Newcomer, The Oxford Classical Monographs, Oxford, Oxford University Press.

VEILLARD C. 2014, «Comment définir son devoir? Les enseignements du plan suivi par Panétius dans son Peri kathekontos », PhilosAnt 14, p. 71-109.

VESPERINI P. 2012, La philosophia et ses pratiques d'Ennius à Cicéron, BEFAR 348, Rome, École française de Rome.

- 2015, «Le sens d'humanitas à Rome », MEFRA 127, 1 [en ligne]. URL : http://

journals.openedition.org/mefra/2768 [consulté le 4 janvier 2018] ; DOI : 10.4000/mefra.2768.

\section{NOTES}

1. SCHIAVONE 2008, p. 69.

2. IACOBINI 2015, p. 1.

3. BRUNT 1979, p. 62-63.

4. ORESTANO 1967, p. 136-137.

5. SCHIAVONE 2008, p. 276-277.

6. MOATTI 1997, p. 42.

7. SCHIAVONE 2005, p. 77 et 174.

8. Ducos 1996.

9. DUCos 1996, p. 136.

10. CUF, II, p. 67.

11. Cicéron, Leg., I, V-17 (CUF, p. 10).

12. Cicéron, Leg., I, XV-42 (CUF, p. 23-24).

13. SCHIAVONE 2008, p. 258-264 et 363-386.

14. Sur ces notions, nous renvoyons à PALLASSE 1945.

15. PALLASSE 1945, p. 28.

16. MICHEL 2003.

17. La récente édition commentée de $\mathrm{T}$. Reinhardt propose une synthèse sur cette question (Oxford Classical Monographs, 2003, p. 177-181). 
18. Sur l'exil de Cicéron, voir HABICHT 2013, p. 67-98. Voir également VESPERINI 2012, p. 379-390.

19. Fox 2007, chap. 2 : «Struggle, Compensation, and Argument in Cicero's Philosophy ».

20. FOX 2007, p. 41.

21. Cicéron, Traité du destin, texte établi et trad. par A. Yon, 1933, p. V-VI.

22. CUF, p. 7-8.

23. CUF, p. 105.

24. CUF, p. 114.

25. Nous empruntons cette formulation à LADMIRAL 1994, p. 112.

26. VAN DER BLOM 2010, p. 29-34.

27. FOX 2007, p. 23.

28. FOX 2007, p. 26.

29. Cicéron, Rep., I, II-2 (CUF, I, p. 195).

30. VESPERINI 2012, p. 392.

31. Cicéron, Diu., II, II-7 (G.F., p. 200-201).

32. FOX 2007, p. 38.

33. G.F., p. 198-199. M. Fox souligne l'exagération ironique (« ironically exaggerated») de la fin du passage, correctif utile à la volonté forte de voir la vision la plus optimiste d'une éducation philosophique présentée comme définitive dans le De Officiis.

34. VAN DER BLOM 2010, p. 311-324.

35. La Roue à livres, p. 5.

36. FEUVRIER-PREVOTAT 1985.

37. Cicéron, N.D., I, III-6 (La Roue à livres, p. 4).

38. Cicéron, N.D. (La Roue à livres, p. XIII).

39. VESPERINI 2012, p. 390-391.

40. Cicéron, Rep., I, IV-7 (CUF, I, p. 199).

41. Pour une vue d'ensemble, voir FEDELI 1973, en particulier p. 361-376.

42. Nous renvoyons à POHLENZ 1934.

43. Nous renvoyons à VALENTE 1956.

44. FERRARY 1990, p. 2. Voir également FEDELI 1973, p. 408-421.

45. FEDELI 1973, p.361-371. Cette prééminence de Panétius ne doit pas faire oublier l'influence d'Antiochus d'Ascalon, particulièrement sensible dans le De Officiis, voir GRILLI 1975.

46. GABBA 1979, p. 129-130.

47. GRIMAL 1989, p. 3.

48. AUVERLOT 1990, p. 3.

49. AUVERLOT 1990, p. 3.

50. GRIMAL 1989, p. 9.

51. PÖSCHL 1987, p. 340. 
52. Cicéron, Tusc., II, 13 (CUF, p. 84 : «Un champ, si fertile soit-il, ne peut être productif sans culture, et c'est la même chose pour l'âme sans enseignement [...] La culture de l'âme (cultura animi), c'est la philosophie : c'est elle qui extirpe radicalement les vices, met les âmes en état de recevoir les semences, et, pour ainsi dire, sème ce qui, une fois développé, jettera la plus abondante des récoltes. »

53. Président de la Société Internationale des Amis de Cicéron < http:// www.tulliana.eu>. La SIAC est une société savante dédiée à l'étude de Cicéron et de la pensée romaine dans tous les domaines : la philosophie, la littérature, l'histoire, les faits de civilisation et l'héritage. Son projet comporte aussi une dimension pédagogique et culturelle.

54. ROUSSELOT 2012.

55. LÉVY 2008, p. 10.

56. TESTARD 1962. L'hypothèse envisagée par M. Testard selon laquelle Octave a pu être également le destinataire du De officiis ne paraît pas acceptable, voir FEDELI 1973, p. 358-360.

57. Cicéron, Off., I, 1 (CUF, I, p. 104).

58. Cicéron, Off., II, 45 (CUF, II, p. 37-38).

59. Cicéron, Off. (Classiques en poche, p. XVII).

60. VEILLARD 2014, p. 106-107.

61. Cicéron, Off. (Classiques en poche, p. XVII).

62. Cicéron, Inu., II, LIII-161 (CUF, p. 225).

63. Sur cette notion romaine spécifique et sa signification chez Cicéron, voir PösCHL 1987, p. 346-347.

64. Cicéron, Off., I, VII-20 à 23 (CUF, I, p. 114-115).

65. Ducos 1984, p. $324 s q$.

66. GOURINAT 2016.

67. GOURINAT 2016, p. 257.

68. LÉVY 1992, p. 285-286.

69. LÉVY 1992, p. 284.

70. GOURINAT 2016, p. 258.

71. CUF, I, p. 108.

72. CUF, II, 1930, p. 39.

73. GOURINAT 2016, p. 261.

74. GOURINAT 2016, p. 262.

75. GOURINAT 2016, p. 268.

76. LÉVY 1992, p. 288.

77. GARCEA 2008, p. 105-106.

78. PROST 2008, p. 114.

79. LÉVY 1989, p. 11.

80. LÉVY 1989, p. 12.

81. COLOTTE 2014. 
82. GRIMAL 1989 , p. 5.

83. NARDUCCI 1989, p. 114.

84. VAN DER BLOM 2010, p. 337.

85. FOX 2007, p. 110.

86. DAVID 1980, p. 67.

87. MOATTI 1988, p. 387.

88. CIZEK 1988, p. 16-17.

89. CIZEK 1988, p. 25.

90. CUF, p. 101.

91. Cicéron, Top. 41-45, en particulier 44-45 (CUF, p. 78-79).

92. Cicéron, de Orat., III, 204-205 (CUF, p. 85). Cicéron en évoque l'emploi à propos de l' ornatio.

93. GUARD 2007, p. 50 .

94. GUARD 2007, p. 51.

95. Cicéron, Off., I, 118 (CUF, I, p. 165-166).

96. Cicéron, Off., III, 99-110 (CUF, II, p. 124-131).

97. GUARD 2007, p. 55. L'auteur reprend une citation de MARCHAL 1987.

98. I, 36 ; I, 37 ; I, 79 ; I, 104 ; I, 151 ; II, 89 ; III, 1 ; III, 16 ; III, 104.

99. HERMAND 2009.

100. Cicéron, Off., I, 36-37 (CUF, I, p. 122-123).

101. DYCK 1996, p. 145.

102. DYCK 1996, p. 482.

103. Cicéron, Off., III, 14 (CUF, II, p. 77) : «De fait, ces devoirs dont nous dissertons dans ces livres, les stoïciens les appellent « moyens »; ce sont des devoirs courants et d'une application étendue : beaucoup y atteignent, du fait et d'un bon nature et du progrès dans l'étude (progressione discendi) ».

104. FORSCHNER 2005, p. 298 sq. Voir également LÉVY 2003.

105. NÉDONCELLE 1948.

106. FORSCHNER 2005, p. 302.

107. FORSCHNER 2005, p. 304.

108. FORSCHNER 2005, p. 305.

109. GARBARINO 1980, en particulier p. 198.

110. CUF, p. 228.

111. CUF, 2002, p. 38

112. ERNOUT, MEILLET 1967, p. 277.

113. Cicéron, Off., I, 121 (CUF, I, p. 167).

114. CUF, I, p. 143. Cicéron énonce la même idée en III, 6 (CUF, II, p. 72-73).

115. Cicéron, Off., II, 31-51 (CUF, II, p. 29-42).

116. CUF, II, p. 38

117. Cicéron, Off., I, 80 (CUF, I, p. 144). 
118. Cicéron, Off., II, 48 (CUF, II, p. 39).

119. Cicéron, Fin., I, 59 (CUF, I, p. 39).

120. CUF, II, p. 36.

121. Cicéron, Off., II, 43 (CUF, II, p. 36).

122. CUF, p. 67.

123. CUF, p. 70.

124. AUVRAY-ASSAYAS 2006, p. 87.

125. TATAKIS 1931, p. 194-196.

126. POHLENZ 1934.

127. Platon, R., VII, 520c (Euvres complètes, sous la dir. de L. Brisson, Paris, Flammarion, 2008, p. 1685).

128. Le fait que la connaissance soit expédiée en deux pages au début du De officiis n'est pas sans faire difficulté. Peut-on définir un système d'éducation qui ne repose pas, au moins en dernière instance, sur une base théorique relative à la connaissance?

129. Cicéron, Off., I, 20 (CUF, I, p. 114) : «Parmi les trois autres divisions, la portée la plus étendue appartient à celle qui maintient le lien social entre les hommes et, pour ainsi dire, la communauté de vie. »

130. JAEGER 1964, p. 139-140 (I, 6 : « La cité-État et son idéal de justice ») : l'invention du mot dikaiosunè (qui ne se trouve pas chez Homère) est liée à l'idéal d'égalité que comporte la dikè (règne de la loi, par opposition à celui des autorités judiciaires qu'exprime le mot thémis). Le sens premier est "droiture », " esprit de justice », mais « une fois le nomos [...] codifié, l'idée générale de droiture prit un sens concret: elle signifiera l'obéissance aux lois de l'État, tout comme la « vertu chrétienne consistera à observer les commandements de Dieu ».

131. ATKINS 1990, p. 281.

132. Cicéron, Inu., II, 65 (CUF, p. 176).

133. Cicéron, Leg., II, 23 (CUF, p.52): leges, quae genus illud optumum rei publicae contineant.

134. Cicéron, Off., III, 28 (CUF, II, p. 84) : «Cette seule vertu en effet est la maîtresse et la reine de toutes les vertus. "

135. MICHEL 1976, p. 93.

136. NICOLET 1976, p. 71-121.

137. DAUBIGNEY 1976, p. 14-71. Voir également Cicéron, Off., II, 73 : « Mais, en premier lieu, il faudra, à celui qui gouvernera l'État, veiller à ce que chacun conserve son bien » (CUF, II, p. 55).

138. Cicéron, Off., II, 78-85 (CUF, II, p. 58-63).

139. CUF, I, p. 213-214.

140. NARDUCCI 1989.

141. MICHEL 1976, p. 92.

142. Sur l'humanisme cicéronien, voir VESPERINI 2015. 


\section{RÉSUMÉS}

Cicéron, cherchant en partie à compenser son effacement politique, propose dans le De Officiis plusieurs principes clés qui fondent son éthique et son humanisme, soulignant en particulier que l'homme n'atteint le decorum que s'il respecte dans la conduite de sa vie la particularité de sa nature. Dans une logique de transmission pédagogique, Cicéron adapte ainsi au monde romain et aux réalités de la cité des concepts issus de la philosophie grecque, mais propose aussi un discours sur les rapports entre l'homme et la cité qu'il veut destiné à tous et universellement valable.

In a troubled period of his life and of Roman political life, Cicero, seeking in part to compensate for his political failure, proposes in the De Officiis key principles that underlie his ethics and his humanism, stressing in particular that man cannot reach decorum if he doesn't respect in the conduct of his life the peculiarity of his nature. In a logic of pedagogical transmission Cicero adapts thus to the Roman world and to the realities of the city concepts coming from the Greek philosophy, but also proposes a discourse on the relations between the man and the city that he wants destined to all and universally valid.

\section{INDEX}

oeuvrecitee Loi des Douze Tables

Keywords : decorum, law, natural law, exemplum, glory, mos maiorum, persona, philosophy, probabile, Roman republic, rhetoric, stoicism

Mots-clés : decorum, droit, droit naturel, exemplum, gloire, mos maiorum, persona, philosophie, probabile, république romaine, rhétorique, stoïcisme

nomsmotscles Antiochus d'Ascalon, Arcesilas, Aristote, Carnéade, Caton l'Ancien, Cicéron, Columelle, Cratippe de Pergame, Démosthène, Diodote, Eschine, Homère, Laelius Sapiens (Caius), Panaitios de Rhodes, Philon de Larissa, Platon, Pline l'Ancien, Posidonius, Sextus Empiricus, Zénon de Cition

\section{AUTEURS}

\section{FRANCK COLOTTE}

Université du Luxembourg 